\title{
Optical and near-IR spectrophotometry of the galaxy NGC 3310
}

\author{
Miriani G. Pastoriza, ${ }^{1}$ Horacio A. Dottori, ${ }^{1}$ Elena Terlevich, ${ }^{2}$ Roberto Terlevich ${ }^{2}$ \\ and Angeles I. Díaz ${ }^{3}$ \\ ' Depto de Astronomia, Instituto de Física - UFRGS, CP 15051, Porto Alegre, 91500, RS, Brazil \\ ${ }^{2}$ Royal Greenwich Observatory, Madingley Road, Cambridge CB3 OEZ \\ ${ }^{3}$ Depto de Física Teórica, C-XI, Universidad Autónoma de Madrid, Cantoblanco, 28049-Madrid, Spain
}

Accepted 1992 June 25. Received 1992 June 25; in original form 1991 September 11

\begin{abstract}
We present spectrophotometric observations in the optical and near-infrared of the nucleus and six $\mathrm{H}_{\text {II }}$ regions in the spiral galaxy NGC 3310. Four of the regions are circumnuclear at less than $400 \mathrm{pc}$ from the nucleus, and two are located in the spiral arms at 600 and $1600 \mathrm{pc}$ respectively. Abundance analysis complemented with detailed photoionization models indicates that, while the circumnuclear and disc $\mathrm{H}_{\text {II }}$ regions have a moderately low metallicity $\left(0.2-0.4 \mathrm{Z}_{\odot}\right)$, the nucleus has solar abundances. No radial gradient was detected away from the nuclear region. The IR $\mathrm{Ca}$ II triplet in absorption is observed in some of the $\mathrm{H}_{\text {II }}$ regions and a moderately strong WR spectral feature at $4686 \AA$ in the brightest $\mathrm{H}_{\text {II }}$ region $9 \operatorname{arcsec} \mathrm{SW}$ of the nucleus is also reported. The simultaneous occurrence of these features in $\mathrm{H}$ II regions with strong emission lines indicates that star formation lasted a few million years.
\end{abstract}

Key words: H II regions - galaxies: abundances - galaxies: individual: NGC 3310 galaxies: peculiar - galaxies: stellar content.

\section{INTRODUCTION}

The possible connection between nuclear activity and star formation has recently received considerable attention and there is a rapidly growing body of evidence suggesting a link, possibly evolutionary, between AGN and starbursts (Terlevich \& Melnick 1985; Terlevich, Melnick \& Moles 1987; Norman \& Scoville 1988). Several so-called hotspot galaxies are composite in nature with a central LINER or Seyfert-like nucleus surrounded by star-forming regions. The central hotspots have high surface brightness, being comparable in luminosity to bright giant $\mathrm{H}_{\text {II }}$ regions. Their most unusual property is a very strong optical continuum relative to the emission-line luminosity. This may reflect a long history of star formation or, alternatively, an unusual stellar mass spectrum in the ionizing cluster (Kennicutt, Keel \& Blaha 1989: KKB). In order to understand the properties of nuclear and extranuclear bursts of star formation, we have embarked on a detailed study of nearby hotspot galaxies of which NGC 3310 is one of the most outstanding examples.

NGC 3310 has been classified as an SAB(r)bc peculiar galaxy of luminosity class II (de Vaucouleurs, de Vaucouleurs \& Corwin 1976). The central part of the galaxy shows a conspicuous 20-arcsec diameter ring of luminous giant $\mathrm{H}$ II regions surrounding the blue compact nucleus. This corre- sponds to $\sim 1.2 \mathrm{kpc}$ at the adopted distance of $12.5 \mathrm{Mpc}$ (Bottinelli et al. 1984). This distinctive feature led Sersic \& Pastoriza (1967) to classify it as a hotspot galaxy. NGC 3310 is included in the catalogue of interacting galaxies (Vorontsov-Velyaminov 1977) and in Arp's (1966) Atlas of Peculiar Galaxies.

The giant $\mathrm{H}$ II regions in the ring have been found to be more luminous than average giant $\mathrm{H}$ II regions, and of low metallicity compared to the inner regions of galaxies with similar Hubble type and luminosity (Heckman \& Balick 1980). The velocity field shows non-circular streaming motions and the dynamical centre seems to be displaced from the nucleus (van der Kruit 1976). The rotation curve near the centre is one of the steepest found among spiral galaxies, giving a central mass of $1 \times 10^{9} \mathrm{M}_{\odot}$ (Grothues \& Schmidt-Kaler 1991: GSK91). Another distinctive feature is the presence of a chain of bright knots at about 1 arcmin to the north-west of the nucleus at PA $\sim 300^{\circ}$, interpreted by Bertola \& Sharp (1984) as a jet formed in an explosive event some $1.8 \times 10^{7}$ yr ago.

Infrared $10-\mu \mathrm{m}$ and $J H K L$ colours indicate that the blue $U B V$ colours and intense radio, ultraviolet and $\mathrm{X}$-ray emission detected in this galaxy can be attributed to a luminous burst of star formation younger than $10^{7} \mathrm{yr}$ (Telesco \& Gatley 1984). Strong near-IR Ca II absorption 
lines, signature of young red supergiants, have been reported in one of the circumnuclear giant $\mathrm{H}_{\text {II }}$ regions (Terlevich et al. 1990).

Radio continuum maps show strong radio sources coincident with the giant $\mathrm{H}$ in regions localized in the inner ring and the arms of the galaxy (van der Kruit \& de Bruyn 1976; Seaquist \& Bignell 1977). The radio emission seems to be stronger than that of a normal spiral.

We have obtained high-quality spatially resolved spectrophotometric data over a wide spectral range (3600-9600 $\AA$ ) which allow us to study the ionization structure of the inner 20 arcsec of NGC 3310 and to deduce the abundances of the most important elements $(\mathrm{O}, \mathrm{S}, \mathrm{N}, \mathrm{Ne}$ and $\mathrm{He})$ as well as the properties of the ionizing star clusters (age, metallicity, number of ionizing stars, etc.).

We present the observations and data reduction in Section 2 and the data analysis in Section 3. The H II region parameters are presented in Section 4. In Section 5 we discuss the WR features in the most luminous $\mathrm{H}$ II region and in Section 6 we model the ionizing stellar associations. Finally, the discussion and main conclusions are presented in Section 7.

\section{OBSERVATIONS AND DATA REDUCTION}

The data were obtained in 1989 March with the Isaac Newton Telescope (INT) at the Roque de Los Muchachos
Observatory using the intermediate dispersion spectrograph (IDS), the 235-mm camera and a blue coated GEC CCD. Two different gratings were used (both with 400 groove $\mathrm{mm}^{-1}$ ) to cover a total spectral range from $3650-9700 \AA$ in five individual spectra of about $1400 \AA$ each. The dispersion of $2.3 \AA$ per pixel with the slit width used for the observations $(1.5$ arcsec) yields a spectral resolution of about $4 \AA$. The size of the CCD along the spatial direction is 400 pixels of $0.7 \mathrm{arcsec}$. The slit was set in three different positions over the central region of the galaxy at $\mathrm{PA} 347^{\circ}, 347^{\circ}$ (10 arcsec off nucleus) and $317^{\circ}$, which are marked in the $\mathrm{H} \alpha$ image of Fig. 1, and called position 1, 2 and 3 respectively in the journal of observations (Table 1).

The data reduction was performed with the IRAF package at IF-UFRGS following standard procedures. The CCD frames were dark current bias subtracted and flat-fielded. The subsequent steps in the reduction were sky subtraction, extinction correction and flux calibration. Sets of increments compressed to one dimension were chosen on the basis of the spatial light distribution along the slit for the different position angles. Since the nebular emission is extended and filled most of the slit, sky subtraction was performed using the outermost increments in every data frame. The spectra were corrected for continuous atmospheric extinction using an extinction curve appropriate for La Palma Observatory. The atmospheric absorption bands present in the infrared

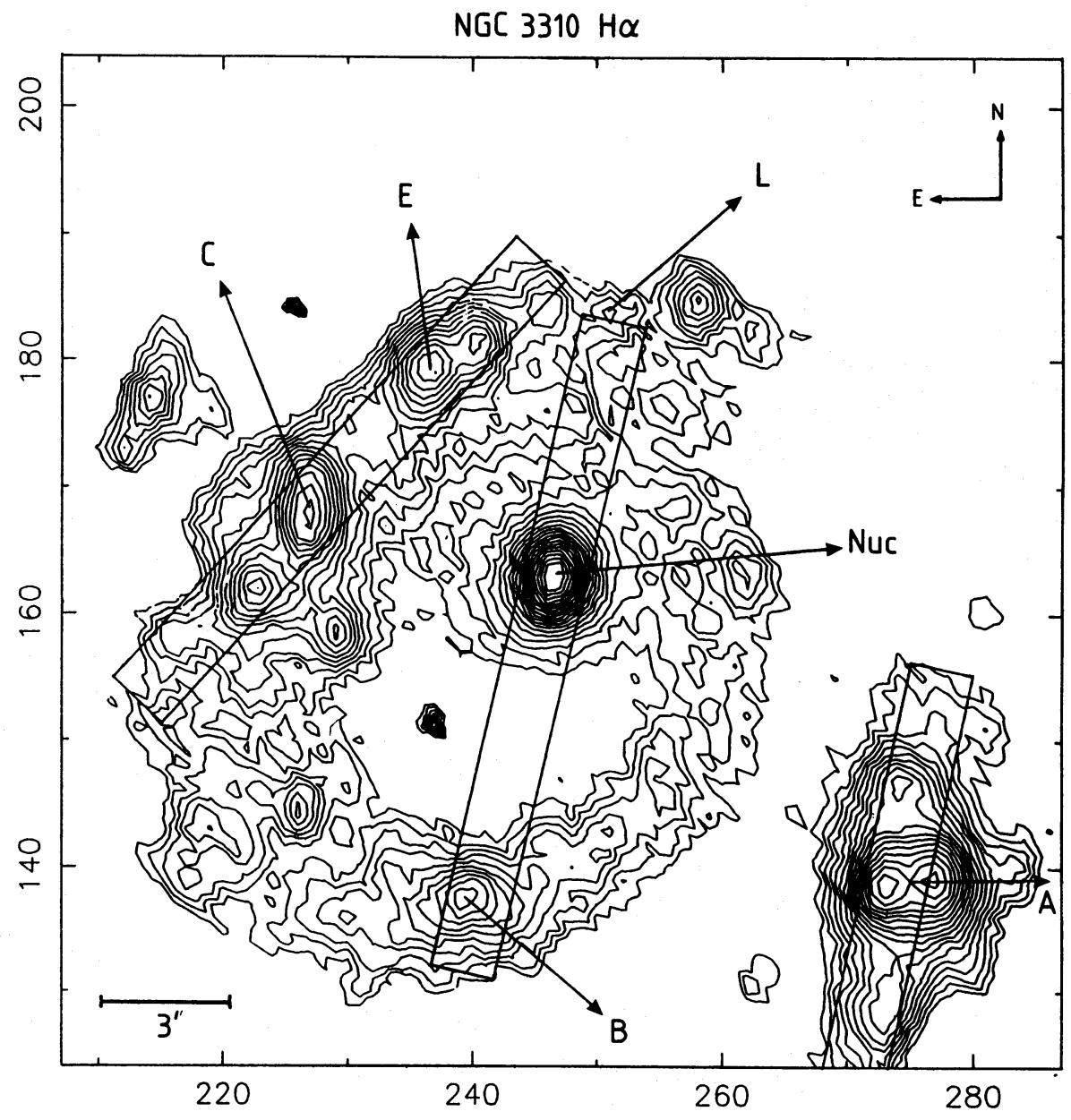

Figure 1. $\mathrm{H} \alpha$ image of NGC 3310 showing our selected slit positions. Nuc, A, B, C, E and L label the regions studied in the abundance analysis. 
Table 1. Journal of observations.

$\begin{array}{cccccc}\text { Position } & \text { Night } & \text { P.A. } & \text { Grating } & \Delta \lambda(\AA) & \text { Exposure (s) } \\ 1 & \text { March 20/21 } & 347 & 400 \mathrm{~V} & 4759-6076 & 1300 \\ 1 & \text { March 20/21 } & 347 & 400 \mathrm{~V} & 4759-6079 & 1300 \\ 1 & \text { March 20/21 } & 347 & 400 \mathrm{~V} & 3581-4953 & 1800 \\ 1 & \text { March 20/21 } & 347 & 400 \mathrm{~V} & 3581-4953 & 1800 \\ 1 & \text { March 20/21 } & 347 & 400 \mathrm{~V} & 5942-7265 & 1000 \\ 1 & \text { March 20/21 } & 347 & 400 \mathrm{~V} & 5942-7265 & 1000 \\ 1 & \text { March 20/21 } & 347 & 400 \mathrm{R} & 7163-8490 & 1300 \\ 1 & \text { March 20/21 } & 347 & 400 \mathrm{R} & 7163-8490 & 1300 \\ 1 & \text { March 20/21 } & 347 & 400 \mathrm{R} & 8359-9691 & 1800 \\ 2 & \text { March 21/22 } & 347 \mathrm{Off} & 400 \mathrm{~V} & 4759-6076 & 1300 \\ 2 & \text { March 21/22 } & 347 \mathrm{Off} & 400 \mathrm{~V} & 4759-6076 & 1300 \\ 2 & \text { March 21/22 } & 347 \mathrm{Off} & 400 \mathrm{~V} & 3581-4953 & 1800 \\ 2 & \text { March 21/22 } & 347 \mathrm{Off} & 400 \mathrm{~V} & 3581-4953 & 1800 \\ 2 & \text { March 21/22 } & 347 \mathrm{Off} & 400 \mathrm{~V} & 5942-7265 & 1300 \\ 2 & \text { March 21/22 } & 347 \mathrm{Off} & 400 \mathrm{~V} & 5942-7265 & 1300 \\ 2 & \text { March 21/22 } & 347 \mathrm{Off} & 400 \mathrm{R} & 8359-9691 & 1800 \\ 2 & \text { March 21/22 } & 347 \mathrm{Off} & 400 \mathrm{R} & 8359-9691 & 1100 \\ 2 & \text { March 21/22 } & 347 \mathrm{Off} & 400 \mathrm{R} & 7163-8490 & 1300 \\ 3 & \text { March 21/22 } & 317 & 400 \mathrm{~V} & 4759-6076 & 1300 \\ 3 & \text { March 21/22 } & 317 & 400 \mathrm{~V} & 4759-6076 & 1300 \\ 3 & \text { March 21/22 } & 317 & 400 \mathrm{~V} & 3581-4953 & 1800 \\ 3 & \text { March 21/22 } & 317 & 400 \mathrm{~V} & 3581-4953 & 1800 \\ 3 & \text { March 22/23 } & 317 & 400 \mathrm{R} & 8359-9691 & 1800 \\ 3 & \text { March 22/23 } & 317 & 400 \mathrm{R} & 8359-9691 & 1800 \\ 3 & \text { March 22/23 } & 317 & 400 \mathrm{R} & 5942-7265 & 1000 \\ 3 & \text { March 22/23 } & 317 & 400 \mathrm{R} & 5942-7265 & 1000 \\ 3 & \text { March 22/23 } & 317 & 400 \mathrm{R} & 7163-8490 & 1300\end{array}$

part of the spectrum have been removed by means of division star (HD 140283) following the procedure described in Díaz, Pagel \& Wilson (1985). Oke \& Gunn (1983) standards HD 84937, HD 102224 and HD 140283 were used for flux calibration.

\section{DATA ANALYSIS}

The spatial distribution of the more relevant emission lines along the slit for the three position angles observed is illustrated in Figs 2-4. These show the structure of the inner region of the galaxy and were used to define the regions from which the spectra have been extracted. These regions are also indicated in Fig. 1. The merged spectra for the nucleus and the circumnuclear $\mathrm{H}$ II regions $\mathrm{B}, \mathrm{C}, \mathrm{E}$ and $\mathrm{L}$ are presented in Figs 5(a)-(e). The figures are plotted with two intensity scales in order to show the weakest lines. The merged spectrum of the off-nuclear giant $\mathrm{H}$ in region A (hereafter 'Jumbo') is shown in Fig. 5(f). Note the broad WR feature seen from 4400 to $4700 \AA$. An enlargement of this part of the spectrum is presented in Fig. $5(\mathrm{~g})$. The most prominent lines have been preliminarily identified as $\mathrm{N}$ III, $\mathrm{Nv},[\mathrm{Fe} \mathrm{III}], \mathrm{He}_{\mathrm{I}}, \mathrm{He}_{\mathrm{II}}$ and [Ar IV]. We will discuss these features in detail in Section 5.

\subsection{Spatial profiles of the emission lines}

An idea of the spatial behaviour of the emission-line intensity ratios $\left[\mathrm{O} \mathrm{II}_{\mathrm{II}}\right] 3727 /[\mathrm{O} \mathrm{III}] 5007,[\mathrm{~S} \mathrm{II}] 6717,6730 /[\mathrm{S} \mathrm{III}] 9532$ and $[\mathrm{N} \mathrm{II}] 6583 / \mathrm{H} \alpha$ can be obtained from the profiles presented in Figs 2-4, where the corresponding pairs have been plotted together for the three slit positions. Due to the wavelength range covered by the line pairs, except for $\left[\mathrm{N}_{\mathrm{II}}\right]$ and $\mathrm{H} \alpha$, these profiles do not represent real excitation differences, as they are affected by reddening.

The ratio $[\mathrm{N} \mathrm{III}] / \mathrm{H} \alpha$ has a constant value of 0.2 for the $\mathrm{H}_{\mathrm{II}}$ regions in positions 2 and 3 , while its value changes from 0.2 to 0.6 along position 1 over the nucleus of the galaxy. This intensity ratio is plotted in Fig. 2(d). The higher value of $[\mathrm{N} \mathrm{II}] / \mathrm{H} \alpha$ in the nucleus is mainly due to an abundance effect, as will be discussed in Section 4.

\subsection{Line intensities}

Line intensities for all the identified emission lines have been measured in each spectrum using the program SPEED (kindly provided by Alex Schmidt), which integrates each line between selected limits and a fitted continuum. It can be seen in the spectra (Figs 5a, b and c) that the Balmer emission lines for most of the observed regions are affected by underlying absorption. We have corrected the emission lines by estimating the equivalent width of the absorption lines from the observed absorption profile. Errors have been estimated as the measured statistical noise of the continuum near the lines, multiplied by the FWHM of the line and divided by the intensity of the line. The observed line-intensities listed in Table 2 have been corrected for reddening according to the standard reddening curve (Whitford 1958) and assuming theoretical values for the Balmer decrement corresponding to case B recombination (Brocklehurst 1971). Due to the underlying absorption problem, we have used only the $\mathrm{H} \alpha$ / $\mathrm{H} \beta$ ratio to deduce the reddening constant $C(\mathrm{H} \beta)$, also given in Table 2. The agreement between the theoretical and reddening-corrected observed values for the ratio of Paschen lines to $\mathrm{H} \alpha$ indicates that underlying absorption and reddening have been properly corrected. The $\mathrm{A}_{V}$ does not vary significantly over the observed circumnuclear starburst regions, the largest extinction being observed at the bright nucleus $\mathrm{A}_{V}=1.13$. The Balmer extinction values obtained in this paper are lower than those found by GSK91.

In Table 2 we have also included the emission-line intensities of an extranuclear $\mathrm{H}$ II region $(\mathrm{M})$, located at 30 $\operatorname{arcsec} \mathrm{N}$ from the nucleus along slit position 1, in order to compare its physical properties with those of the circumnuclear regions. The continuum fluxes at $4000,4678,5510$ and $8500 \AA$ are listed in Table 3.

\subsection{Gas densities and temperatures}

Gas densities, obtained from the $\left[\mathrm{S}_{\mathrm{II}}\right] 6717,6731 \AA$ line ratios (Osterbrock 1989), have been found to be of the order of $200 \mathrm{~cm}^{-3}$ for the Hil regions and $8000 \mathrm{~cm}^{-3}$ for the nucleus. Four line ratios were used for temperature determination: $[\mathrm{O}$ III $] 4363 /(5007+4959) \quad \AA,[\mathrm{O}$ II $](7320+7330) /$ $3727 \AA,\left[\mathrm{S}_{\mathrm{III}}\right] 6312 /(9069+9532) \AA$ and $\left[\mathrm{S}_{\mathrm{II}}\right](6717+$ $6730) /(4068+4076) \AA$, following the relations proposed by Seaton (1975) and Aller (1984) with the atomic parameters given by Mendoza (1983). The derived values are listed in Table 4. Differences of temperature larger than $1000 \mathrm{~K}$ for different ions in each $\mathrm{H}_{\text {II }}$ region are compatible with the flux errors of the weakest lines. We have adopted for the $\mathrm{H}$ II regions $\mathrm{B}, \mathrm{C}$ and $\mathrm{E}$ the temperatures of $[\mathrm{O} \mathrm{II}]$ and $[\mathrm{O} \mathrm{III}]$ for low- and high-ionization zones respectively, because they 

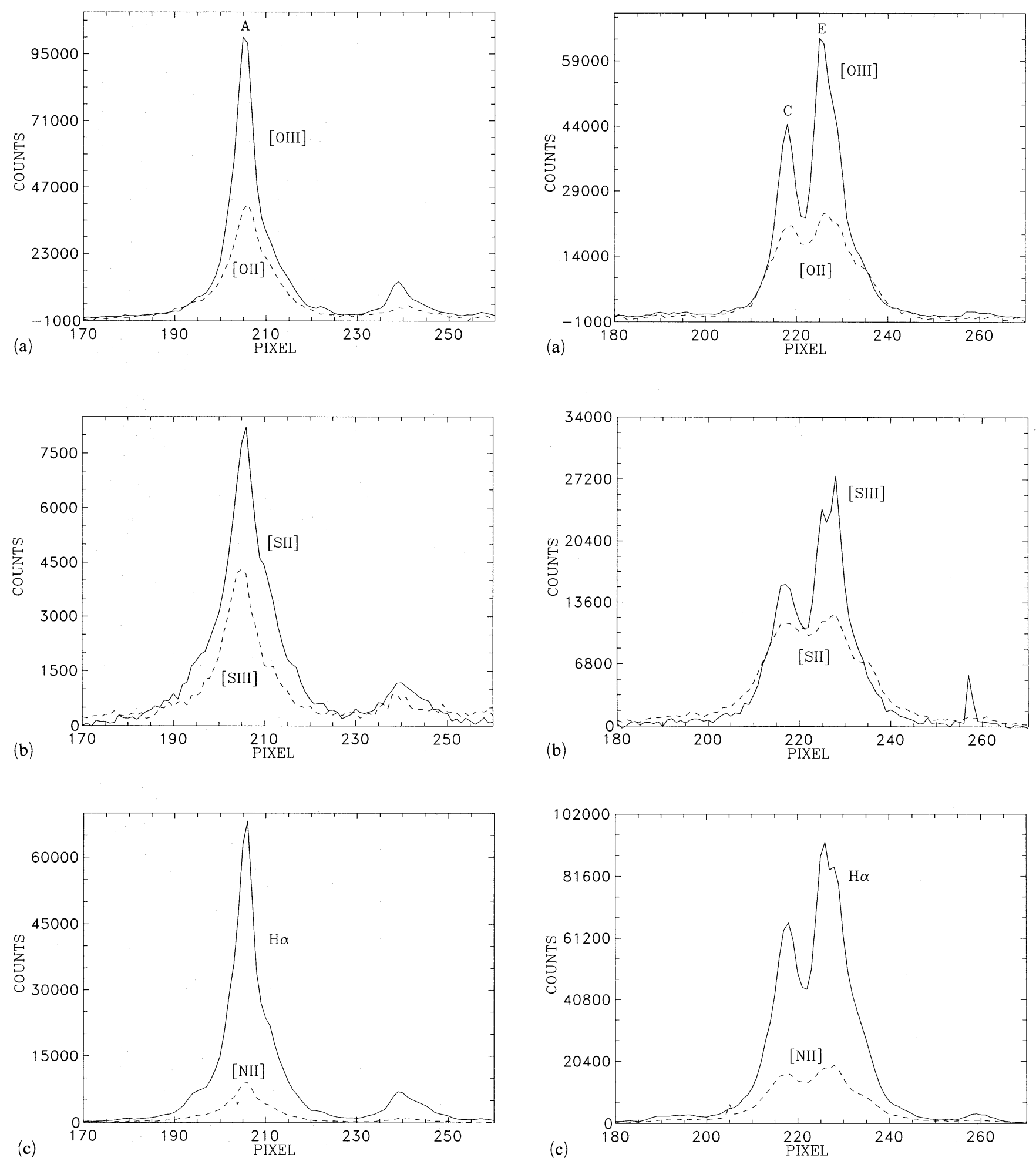

Figure 3. (a)-(c) Spatial profiles of the same emission lines as in Fig. 2, along region $\mathrm{A}$.

density of $100 \mathrm{~cm}^{-3}$ (appropriate for the circumnuclear regions) and abundances $Z=0.1,0.2,0.4$ and $0.8 \mathrm{Z}_{\odot}$ have been used to calculate the models.

Fig. $6($ a) shows $\log ([\mathrm{O} \mathrm{III}] / \mathrm{H} \beta)$ versus $\log ([\mathrm{S} \mathrm{II}] / \mathrm{H} \alpha)$ for the computed models. The ratio $[\mathrm{S} \mathrm{II}] / \mathrm{H} \alpha$ is strongly dependent

on $U$ (Evans \& Dopita 1985 ), while $[\mathrm{O} \mathrm{III}] / \mathrm{H} \beta$ is sensitive to the $T_{\text {eff }}$ of the ionizing stars and the metallicity of the gas. Fig. $6(\mathrm{~b})$ shows the model trends for another good $U$ indicator $\log \left(\left[\mathrm{S}_{\mathrm{II}}\right] /\left[\mathrm{S}_{\mathrm{III}}\right]\right)$ as plotted against $\log \left(\left[\mathrm{O}_{\mathrm{III}}\right] / \mathrm{H} \beta\right)$. Comparison with the $\mathrm{H}$ II region data indicates that values of $\log U \sim-3$

Figure 4. (a)-(c) Spatial profiles of the same emission lines as in Fig. 2, for regions $\mathrm{C}$ and $\mathrm{E}$. 

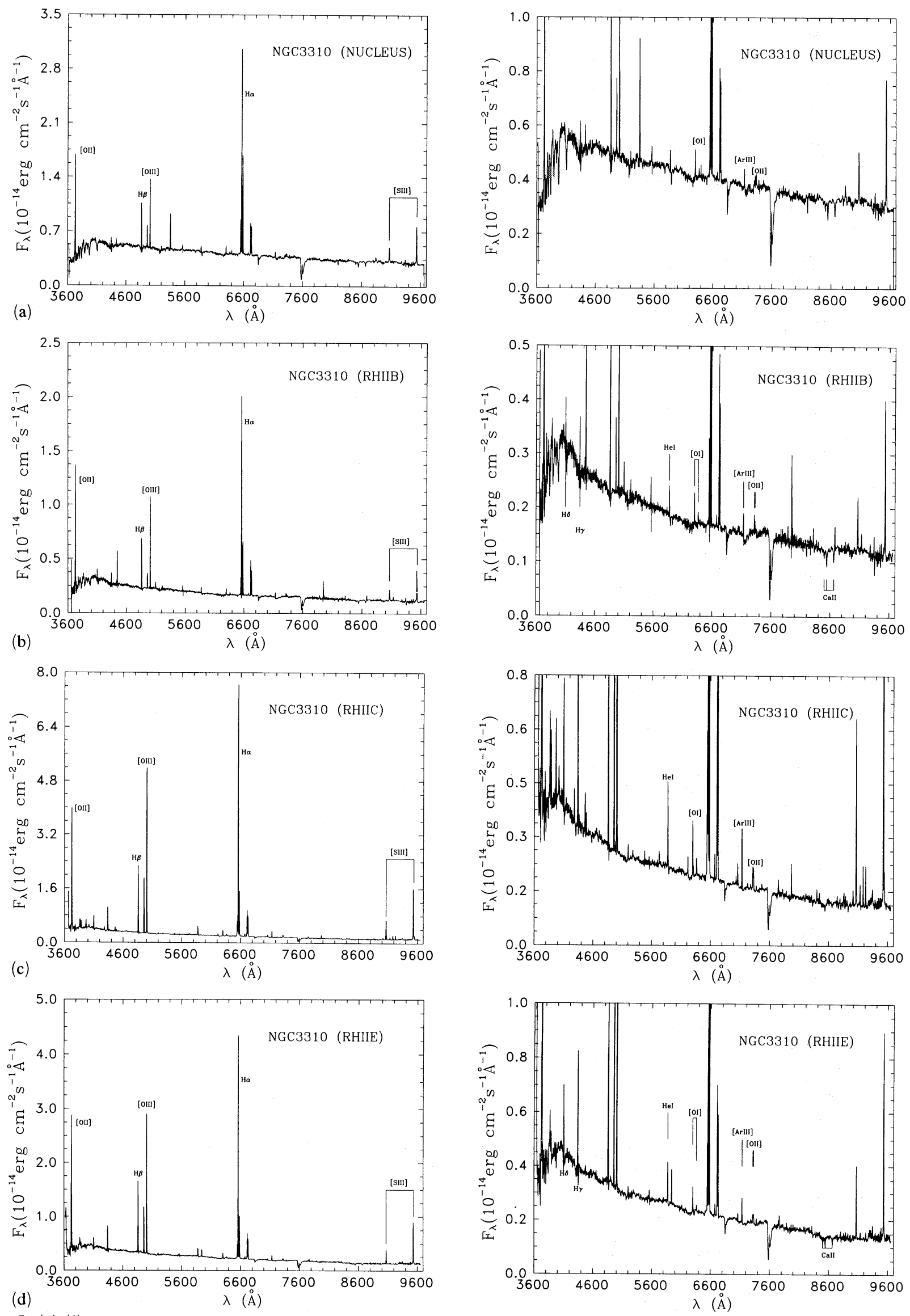

Figure 5. (a)-(f) Merged spectrum with two intensity scales for the nucleus and circumnuclear regions A, B, C, E and L. Notice the WR feature at $4600 \AA$. (g) An enlargement of the WR features due to WN4-5 stars in the Jumbo (A) region. 
Table 2. Fluxes for the $\mathrm{H}$ II regions.

\begin{tabular}{|c|c|c|c|c|c|c|c|}
\hline $\begin{array}{c}\text { Region } \\
\text { Line }\end{array}$ & Nuc & $\mathbf{A}$ & B & $\mathrm{C}$ & $\mathbf{E}$ & L & M \\
\hline 3727 [OII] & $403 \pm 1$ & $259 \pm 4$ & $311 \pm 7$ & $316 \pm 10$ & $313 \pm 4.5$ & $469 \pm 19$ & $251 \pm 13$ \\
\hline 3869 [NeIII] & - & $18 \pm 4$ & $16 \pm 4$ & $19 \pm 3$ & $24 \pm 4.4$ & $9.5 \pm 1$ & 13 \\
\hline $3889 \mathrm{H} 8, \mathrm{HeI}$ & - & $13 \pm 3.9$ & $100 \pm 2$ & $15 \pm 4$ & $13 \pm 4.3$ & - & - \\
\hline 3967 [NeIII], H $\epsilon$ & - & $17 \pm 5.3$ & $9 \pm 2$ & $16 \pm 3$ & - & - & - \\
\hline $4068,76[\mathrm{SII}]$ & $17 \pm 7$ & $4.2 \pm 1.1$ & 4: & 4.4: & - & - & - \\
\hline $4101 \mathrm{H} \delta$ & $10 \pm 6$ & 21: & $21 \pm 1$ & $25 \pm 2$ & $19 \pm 4$ & $22 \pm 0.4$ & 33 \\
\hline $4340 \mathrm{H} \gamma$ & $29 \pm 9$ & 39.5: & $38 \pm 1$ & $43 \pm 2$ & $40 \pm 4$ & $40 \pm 3$ & 39 \\
\hline 4363 [OIII] & - & $1.8 \pm 0.5$ & $2:$ & $2.5 \pm 0.7$ & $1.2:$ & - & - \\
\hline $4471 \mathrm{HeI}$ & - & $3.6 \pm 0.9$ & 3: & $3.3 \pm 1.2$ & $3 \pm 1$ & - & - \\
\hline 4672 FeIII & - & 16: & - & - & - & - & - \\
\hline $4861 \mathrm{H} \beta$ & $100 \pm 4$ & $100 \pm 5.3$ & $100 \pm 0.8$ & $100 \pm 1$ & $100 \pm 2.2$ & $100 \pm 4$ & 100 \\
\hline 4959 [OIII] & $43 \pm 4$ & $74 \pm 0.7$ & $59 \pm 1$ & $81 \pm 1$ & $69 \pm 0.1$ & $57 \pm 4$ & $91 \pm 20$ \\
\hline 5007 [OIII] & $134 \pm 3$ & $231 \pm 0.9$ & $182 \pm 1$ & $245 \pm 1.2$ & $202 \pm 1.4$ & $168 \pm 4$ & $249 \pm 8$ \\
\hline $5012 \mathrm{HeI}$ & - & $1.5 \pm 0.6$ & - & - & - & - & - \\
\hline $5198[\mathrm{NI}]$ & $8.6 \pm 3.6$ & $1.5 \pm 0.3$ & 3: & $2.3 \pm 0.7$ & $4 \pm 1$ & - & - \\
\hline $5270[\mathrm{FeI}]$ & - & $0.9:$ & - & - & - & - & - \\
\hline $5755[\mathrm{NII}]$ & - & 0.4: & - & - & - & - & - \\
\hline $5876 \mathrm{HeI}$ & $10 \pm 1$ & $9.9 \pm 0.5$ & $13 \pm 1$ & $11 \pm 0.6$ & $11 \pm 1.1$ & $17 \pm 1$ & - \\
\hline $6300[\mathrm{OI}]$ & $12 \pm 1$ & $5.1 \pm 0.4$ & $6 \pm 1$ & $6.5 \pm 0.4$ & $7 \pm 1$ & $9.6 \pm 0.9$ & - \\
\hline 6312 [SIII] & - & $1.1 \pm 0.3$ & - & $1.4 \pm 0.2$ & $1.4 \pm 0.7$ & $1.1 \pm 0.5$ & - \\
\hline 6364 [OI] & 23 & $1.4 \pm 0.5$ & $2:$ & $2.3 \pm 0.3$ & $2.2 \pm 0.7$ & $2.7 \pm 0.1$ & 7 \\
\hline 6548 [NII] & $48.5 \pm 2$ & $15 \pm 0.5$ & $21 \pm 0.9$ & $18 \pm 0.3$ & $20 \pm 0.9$ & $26 \pm 1.1$ & 18 \\
\hline $\begin{array}{c}6563 \mathrm{H} \alpha \\
6584 \text { [NII] }\end{array}$ & $\begin{array}{l}290 \pm 2 \\
146+2\end{array}$ & $\begin{array}{c}288 \pm 0.4 \\
46+0.3\end{array}$ & $287 \pm 0.9$ & $\begin{array}{c}288 \pm 0.4 \\
55+0.4\end{array}$ & $\begin{array}{c}287 \pm 1.2 \\
59+0.7\end{array}$ & $289 \pm 1.6$ & $\begin{array}{l}287 \pm 5 \\
43+10\end{array}$ \\
\hline $6678 \mathrm{HeI}$ & $3 \pm 1$ & $3.2 \pm 0.3$ & $3 \pm 0.6$ & $3.5 \pm 0.3$ & $5 \pm 0.7$ & $3.6 \pm 0.8$ & - \\
\hline 6717 [SII] & $47 \pm 2$ & $27 \pm 0.6$ & $39 \pm 0.7$ & $31 \pm 0.2$ & $35 \pm 2$ & $48 \pm 0.9$ & $34 \pm 10$ \\
\hline $6730[\mathrm{SII}]$ & $88 \pm 2$ & $22 \pm 0.6$ & $31 \pm 0.7$ & $24 \pm 0.2$ & $27 \pm 0.7$ & $37 \pm 1.1$ & $27 \pm 10$ \\
\hline $7065 \mathrm{HeI}$ & - & - & $1.9 \pm 0.6$ & $2 \pm 0.2$ & $1 \pm 0.7$ & - & - \\
\hline 7137 [ArIII] & $5.7 \pm 1.1$ & $0.7:$ & $5 \pm 0.6$ & $7.7 \pm 0.2$ & $6.7 \pm 0.7$ & $7.5 \pm 1.1$ & 10 \\
\hline $7319,30[\mathrm{OII}]$ & $13 \pm 3$ & $0.7:$ & $7 \pm 1$ & $5.5 \pm 0.5$ & $6.6 \pm 0.7$ & $6.3 \pm 2.1$ & 8 \\
\hline 7750 [ArIII] &.- & - & - & $1.9 \pm 0.3$ & 2.2: & - & - \\
\hline 8446 FeII & - & 0.6 & - & - & - & - & - \\
\hline 8598 P14 & - & 0.3 & - & - & - & - & - \\
\hline 8750 P12 & - & 0.3 & - & - & - & - & - \\
\hline 8863 P11 & - & 0.7 & - & - & - & - & - \\
\hline 9015 P10 & - & 1 & $3 \pm 0.6$ & $1.9 \pm 0.2$ & - & - & - \\
\hline 9069 [SIII] & $23 \pm 1.3$ & $12 \pm 0.4$ & $22 \pm 0.7$ & $24 \pm 0.6$ & $22.2 \pm 1.6$ & $18 \pm 1.2$ & $21 \pm 10$ \\
\hline 9229 P9 & - & $1.5 \pm 0.3$ & $2 \pm 0.7$ & $5 \pm 0.6$ & - & - & - \\
\hline 9532 [SIII] & $74 \pm 1.3$ & $31 \pm 0.3$ & $39 \pm 1$ & $57 \pm 0.3$ & $54 \pm 0.8$ & $49 \pm 2.3$ & $58 \pm 10$ \\
\hline $9556 \mathrm{P} 8$ & - & $2.1 \pm 0.2$ & - & $4.6 \pm 0.3$ & - & - & - \\
\hline $\mathrm{C}(\mathrm{H} \beta)$ & 0.63 & 0.29 & 0.2 & 0.34 & 0.26 & 0.53 & 0.14 \\
\hline $\mathrm{F}(\mathrm{H} \beta)$ & 3.6 & 16.9 & 5.9 & 13.2 & 7.1 & 2.6 & 1.0 \\
\hline $\mathrm{EW}(\mathbf{H} \beta)(\AA)$ & 12 & 73 & 29 & 55 & 30 & 15 & 38 \\
\hline $\mathrm{L}(\mathrm{H} \beta)$ & 2.7 & 5.7 & 1.6 & 5.0 & 2.3 & 1.5 & 0.2 \\
\hline
\end{tabular}

Adopted distance $=12.5 \mathrm{Mpc} ; \mathrm{F}(\mathrm{H} \beta)$ in $10^{-14} \mathrm{erg} \mathrm{cm}^{-2} \mathrm{~s}^{-1} ; \mathrm{L}(\mathrm{H} \beta)$ in $10^{39} \mathrm{erg} \mathrm{s}^{-1}$.

Table 3. Continuum fluxes for the $\mathrm{H}$ II regions.

$\begin{array}{cccccccc} & \text { Nuc } & \text { A } & \text { B } & \text { C } & \text { E } & \text { L } & \text { M } \\ \lambda 4000 & 0.58 & 0.40 & 0.45 & 0.45 & 0.47 & 0.32 & 0.05 \\ \lambda 4678 & 0.51 & 0.30 & 0.31 & 0.33 & 0.36 & 0.24 & 0.02 \\ \lambda 5510 & 0.45 & 0.20 & 0.24 & 0.25 & 0.28 & 0.20 & 0.03 \\ \lambda 8500 & 0.33 & 0.09 & 0.13 & 0.13 & 0.14 & 0.12 & 0.01\end{array}$

Fluxes in $10^{-14} \mathrm{erg} \mathrm{cm}^{-2} \mathrm{~s}^{-1}$. and $T_{\text {eff }} \sim 40000 \mathrm{~K}$ are consistent in both diagrams. Using this $U$ value, we have constructed abundance-dependent diagrams involving the strongest emission lines observed in the $\mathrm{H}$ II regions (Figs $6 \mathrm{c}$ and $\mathrm{d}$ ). The continuous line in these diagrams connects models with equal abundance. The abundances of nitrogen and oxygen relative to solar, Fig. 6(c), show that the loci of the circumnuclear $\mathrm{H}$ il regions $\mathrm{A}$, $\mathrm{B}, \mathrm{C}, \mathrm{E}$ and $\mathrm{L}$, for $\log U=-3$ and $T_{\text {eff }} \sim 4.0 \times 10^{4}$, fall 
photons of $8.9 \times 10^{51}$ photon $\mathrm{s}^{-1}$ [assuming case $\mathrm{B}$ recombination, with $T_{\text {eff }}=1 \times 10^{4} \mathrm{~K}$ and $N_{\mathrm{e}}=100 \mathrm{~cm}^{-3}$ (Osterbrock 1989)]. As many as $570 \mathrm{O} 6$ stars, each one producing $\log Q=49.23$ photon $\mathrm{s}^{-1}$, are needed to account for the observed $\mathrm{H} \beta$ luminosity. This estimate indicates a WR/O ratio larger than $1 / 3$, comparable to that of 30 Dor (WR/ O 1/7; Melnick 1985; Walborn 1991) considering the recently resolved contents of its ionizing core R136a by the Hubble Space Telescope $(H S T)(\mathrm{WR} / \mathrm{O} \sim 1 / 3)$ (Walborn et al. 1992). These estimates should be taken cautiously. One
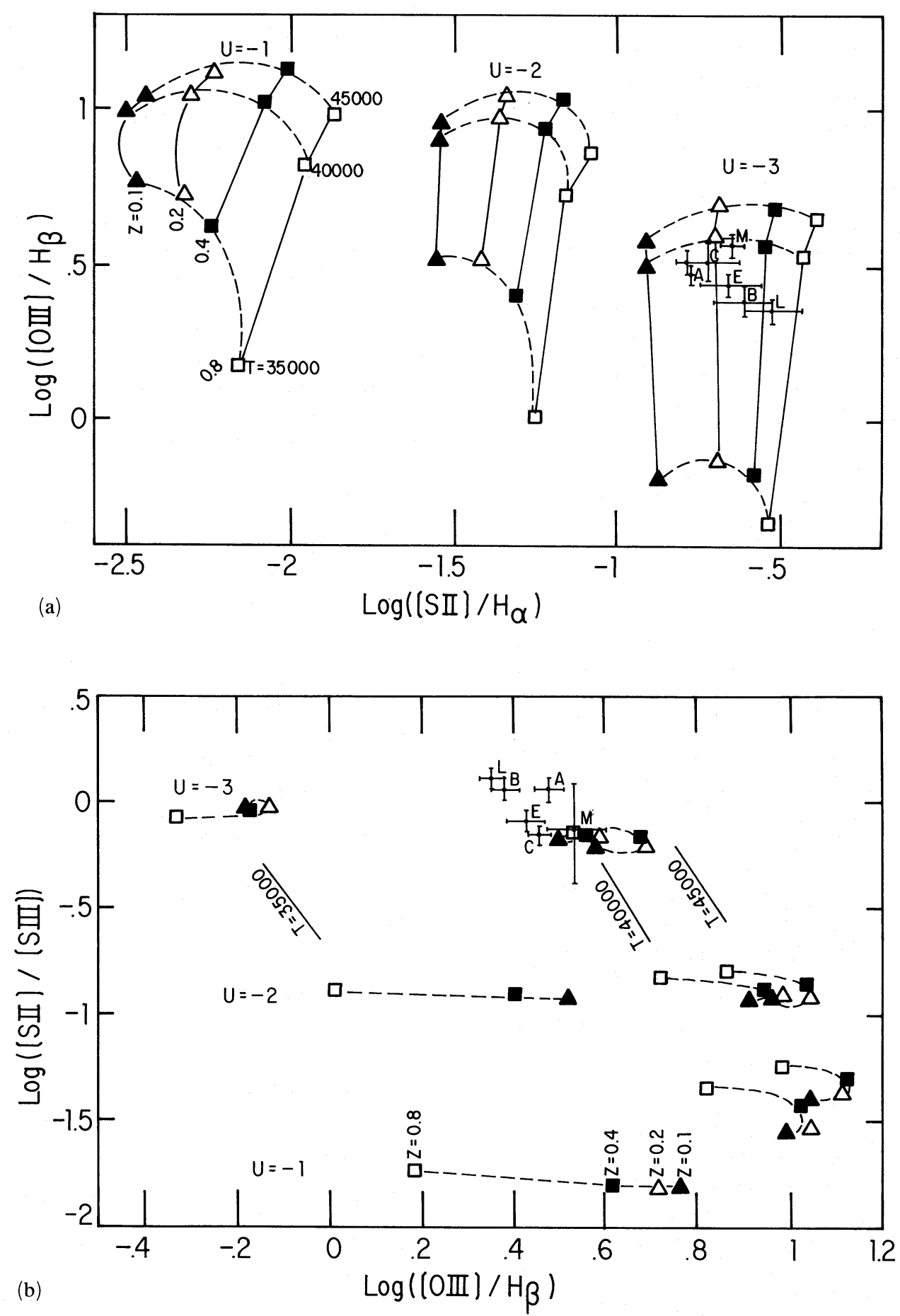

Figure 6. (a) $\left[\mathrm{O}_{\mathrm{III}}\right] / \mathrm{H} \beta$ as a function of $\left[\mathrm{S}_{\mathrm{II}}\right] / \mathrm{H} \alpha$ from the photoionization models described in the text. All the observed regions fall near models with $\log U=-3$ and $T_{\text {eff }}=40000 \mathrm{~K}$. (b) $[\mathrm{S} \mathrm{II}] /[\mathrm{S} \mathrm{III}]$ as a function of $[\mathrm{O} \mathrm{III}] / \mathrm{H} \beta$. This diagram is also $U$ and $T$ dependent. The observed regions are again near models with $\log U=-3$ and $T_{\text {eff }}=40000 \mathrm{~K}$. (c) $\left[\mathrm{N}_{\mathrm{II}}\right] / \mathrm{H} \alpha$ versus $\left(\left[\mathrm{O}_{\mathrm{II}}\right]+\left[\mathrm{O}_{\mathrm{III}}\right]\right) / \mathrm{H} \beta$ from models with $\log U=-3$. This diagram is abundance-dependent; the observed $\mathrm{H}_{\mathrm{II}}$ regions are near the $Z=0.1 \mathrm{Z}_{\odot}$ line. $(\mathrm{d})\left([\mathrm{S} \mathrm{II}]+\left[\mathrm{S}_{\mathrm{III}}\right]\right) / \mathrm{H} \beta$ as a function of $\left(\left[\mathrm{O}_{\mathrm{II}}\right]+\left[\mathrm{O}_{\mathrm{III}}\right]\right) / \mathrm{H} \beta$, also an abundance-dependent diagram, stressing the above conclusions. 

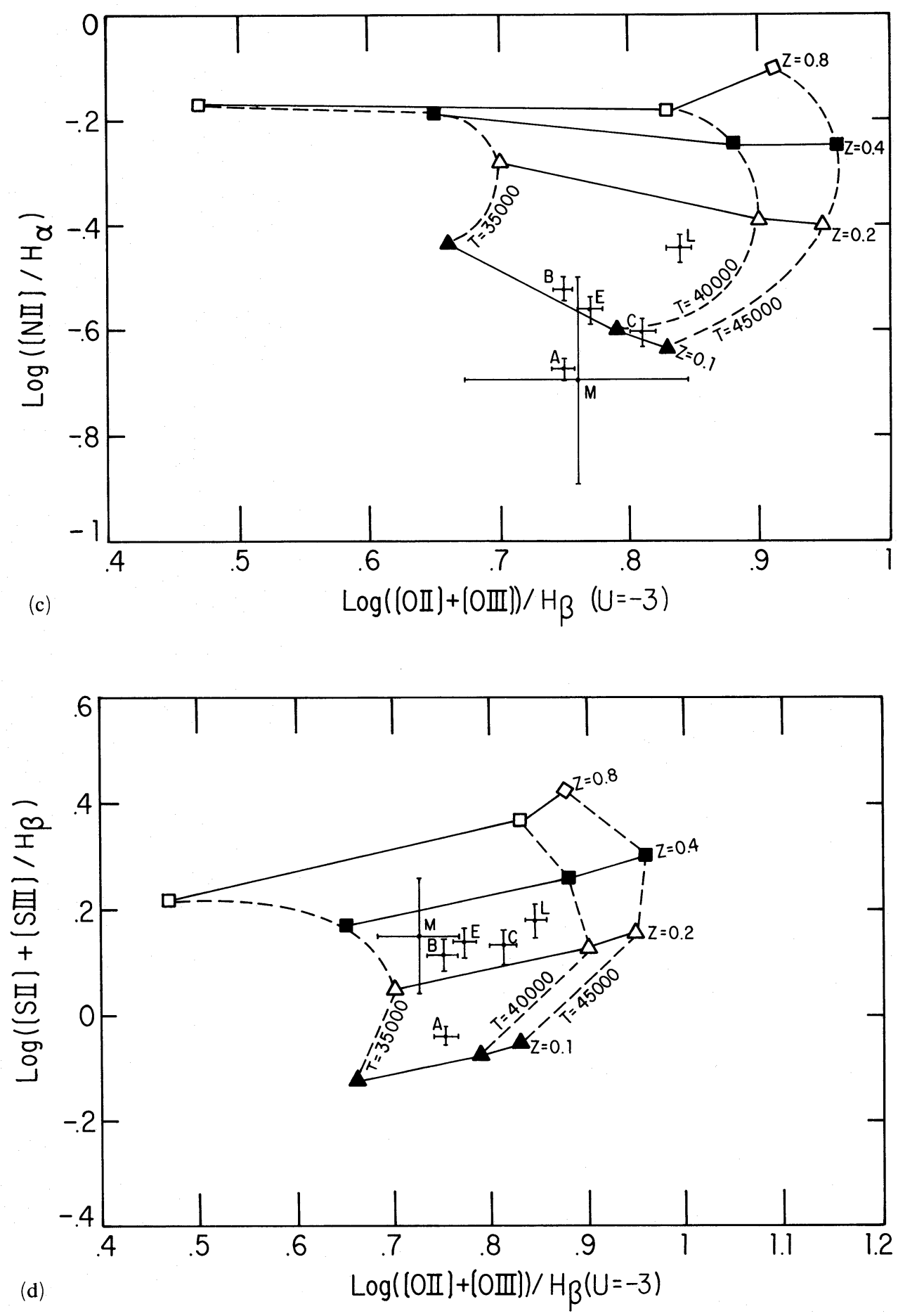

Figure 6 - continued

caveat is the higher spatial resolution of the 30 Dor observations. In our integrated spectra, we might be seeing WR stars that do not belong to the $\mathrm{H}$ II region.

\section{MODELLING THE IONIZING ASSOCIATIONS}

In order to obtain a better insight into the internal properties that govern the process of star formation in $\mathrm{H}$ II regions, we have compared the observations with simple models of ionizing clusters. The models were computed using a code developed by R. Cid-Fernandes (Cid-Fernandes 1989; Cid Fernandes et al. 1992). The code uses the library of evolutionary tracks by Maeder \& Meynet (1989) and Maeder (1990) (for stars between 0.8 and $120 \mathrm{M}_{\odot}$ ) and model atmospheres from Clegg \& Middlemass (1987), Wesemael (1981) and Kurucz (1979).

Several observational constraints are used for the models. 
Table 6. Equivalent width and luminosity of the WR feature.

$\begin{array}{lcccc} & \lambda(\AA) & W_{\text {neb }}(\AA) & W_{\mathbf{s}}(\AA) & \mathrm{L}\left(10^{38} \mathrm{erg} \mathrm{s}^{-1}\right) \\ \text { HeII } & 4686 & 1.2 & 3.9 & 2.2 \\ \text { NIII } & 4634-40 & 1.1 & 1.8 & 1.0 \\ \text { NV } & 4620 & 0.8 & 1.6 & 0.9 \\ \text { NV } & 4603 & 0.7 & 1.0 & 0.6 \\ \text { OII } & 4591 & 0.8 & - & - \\ & {[4550-4582]} & - & 3.1 & - \\ \text { [ArV] } & 4711 & 0.9 & - & - \\ {[\mathrm{FeIII}]} & 4658 & 2.4 & - & - \\ {[\mathrm{ArV}]} & 4625 & 0.7 & - & -\end{array}$

$W_{\text {neb }}$ and $W_{\mathrm{s}}$ correspond respectively to the equivalent widths of the nebular and star components of the line Continuum at $\lambda 4686=4.8 \times 10^{-15} \mathrm{erg} \mathrm{s}^{-1}$.

(i) The continuum colours. Observed continuum fluxes are listed in Table 3; four continuum points at 4000, 4680, 5500 and $8500 \AA$ are included.

(ii) The luminosity and equivalent width of $\mathrm{H} \beta$. These are listed in Table 2.

(iii) The detection of WR features, indicating the presence of evolved massive stars $\left(M \sim 40 \mathrm{M}_{\odot}\right)$.

(iv) The detection of a strong Ca II triplet at $8500 \AA$, indicating that red supergiant (RSG) stars are present (Terlevich et al. 1990). These RSG are associated with evolved intermediate-mass stars $\left(M<20 \mathrm{M}_{\odot}\right)$.

(v) The observed equivalent width of the WR lines.

The equivalent width of the $\mathrm{He}$ II $4686 \AA$ line observed in the Jumbo region is $3 \AA$. The observed value for WR4-5 stars ranges from $80 \AA \leq W_{\mathrm{WR}}(4686) \leq 300 \AA$ (SK81). Thus, the contribution to the continuum at $4686 \AA$ from stars other than the WR must be 25-100 times higher than that of the WR themselves.

We found that the observational constraints cannot be explained simultaneously with a stellar cluster having a single age. Regions A, B, C, E and L can be successfully modelled with two cycles of star formation:

(i) a population $15 \mathrm{Myr}$ old providing the IR continuum and the IR Ca II triplet, and

(ii) a younger population providing the ionizing UV photons.

The cluster parameters (identified as I for the old cluster, II for the young cluster with WR and III for the young cluster without WR) and the derived fluxes for the selected continuum points are listed in Table 7 as well as the total ionizing photons $Q(\mathrm{H})$ and $Q(\mathrm{He})$ emitted by $(1)$ all cluster stars, (2) only the WN stars, and (3) stars of $M>20 \mathrm{M}_{\odot}$. The properties are scaled to a $1-\mathrm{M}_{\odot}$ cluster.

The observed continuum slope, and the $\mathrm{H} \beta$ equivalent width and luminosity of the circumnuclear $\mathrm{H}_{\text {II }}$ regions are well described by a model that has a large contribution $(>70$ per cent) from the older stellar population (cluster I) and a small contribution from the younger one (cluster II). This is illustrated in Fig. 7.

To reproduce the spectral characteristics of region A requires a model with equal proportion of clusters I and II,
Table 7. Elements of the composite clusters.

\begin{tabular}{|c|c|c|c|}
\hline Age Myr & $\begin{array}{c}\mathrm{I} \\
14.5\end{array}$ & $\begin{array}{c}\text { II } \\
5.3\end{array}$ & $\begin{array}{l}\text { III } \\
4.6\end{array}$ \\
\hline IMF $M_{\text {inf }}\left(\mathrm{M}_{\odot}\right)$ & 1 & 1 & 1 \\
\hline IMF $M_{\text {sup }}\left(\mathrm{M}_{\odot}\right)$ & 15 & 40 & 55 \\
\hline Slope & 1.35 & 1.35 & 1.35 \\
\hline Total mass $\left(\mathbf{M}_{\odot}\right)$ & 1 & 1 & 1 \\
\hline N stars & 0.41 & 0.36 & 0.34 \\
\hline $\mathrm{Q}(\mathrm{H})\left(10^{43}\right.$ photons s $\left.{ }^{-1}\right)$ & 6.48 & 1559 & 2823 \\
\hline$F_{c}(\lambda 4000)$ & 5.49 & 28.59 & 62.78 \\
\hline$F_{c}(\lambda 4678)$ & 4.06 & 19.50 & 39.43 \\
\hline$F_{c}(\lambda 5510)$ & 3.23 & 12.12 & 23.44 \\
\hline$F_{c}(\lambda 8500)$ & 1.88 & 3.31 & 5.70 \\
\hline N WNs $\left(10^{-4}\right)$ & - & - & 1.32 \\
\hline $\mathrm{Q}(\mathrm{H})\left(10^{45}\right.$ photons s $\left.^{-1}\right)$ & - & - & 6.74 \\
\hline$F_{c}(\lambda 4000)$ & 一 & - & 1.31 \\
\hline$F_{c}(\lambda 4678)$ & - & - & 0.75 \\
\hline$F_{c}(\lambda 5510)$ & - & - & 0.42 \\
\hline$F_{c}(\lambda 8500)$ & - & - & 0.09 \\
\hline N $M>20\left(10^{-3}\right)$ & - & 3.68 & 4.02 \\
\hline $\mathrm{Q}(\mathrm{H})\left(10^{46}\right.$ photons $\left.\mathrm{s}^{-1}\right)$ & - & 1.45 & 2.06 \\
\hline$F_{c}(\lambda 4000)$ & - & 5.36 & 12.44 \\
\hline$F_{c}(\lambda 4678)$ & - & 3.00 & 7.36 \\
\hline$F_{c}(\lambda 5510)$ & - & 1.67 & 4.26 \\
\hline$F_{c}(\lambda 8500)$ & - & 0.33 & 0.93 \\
\hline
\end{tabular}

Fluxes in $10^{-13} \mathrm{erg} \mathrm{cm}^{-2} \mathrm{~s}^{-1}$.

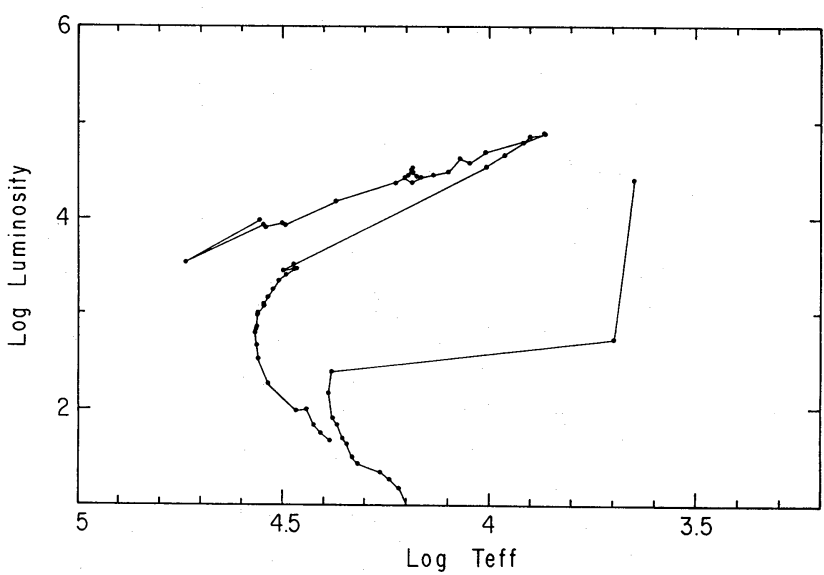

Figure 7. Synthesized two-bursts HR diagram for region A where WR stars are observed.

the last one providing the WR stars. Besides the relative contribution of both components, we can derive from the models the mass of the old and young components and the mass and number of ionizing stars and WN stars. These data are listed in Table 7 for the circumnuclear regions and in Table 8 for region A. Fig. 7 shows the HR diagram of the synthesized clusters for region A. Maeder's models require around $60 \mathrm{WN}$ stars of $50-55 \mathrm{M}_{\odot}$ to account for the WR feature in this region. It is expected that stars up to 35-40 $M_{\odot}$ will go through WR phases for a region of such metallicity. The number of WN would then be increased by a factor of three, in agreement with the previous rough derivation of the number of WN4-5 contained in this region. 
Table 8. Cluster mass of circumnuclear $\mathrm{H}$ in regions.

$\begin{array}{ccccc} & \text { Mass } & \mathrm{B} & \mathrm{C} & \mathrm{E} \\ \text { Proportion } & \text { Cluster I } & 0.8 & 0.7 & 0.8 \\ \text { Proportion } & \text { Cluster II } & 0.2 & 0.3 & 0.2 \\ & M_{\text {old }}\left(10^{6} \mathrm{M}_{\odot}\right) & 1.0 & 1.0 & 1.2 \\ & M_{\text {young }}\left(10^{4} \mathrm{M}_{\odot}\right) & 6.8 & 7.5 & 7.8 \\ & M_{\text {is }}(\mathrm{M}>\mathbf{2 0 M} \odot) & 2000 & 7000 & 3200 \\ & N_{\text {is }(\mathrm{M}=30 \mathrm{M} \odot)} & 70 & 240 & 106\end{array}$

The penultimate row gives the total mass of ionizing stars with mass $\geq 20 \mathrm{M}_{\odot}$. The last row gives the total number of ionizing stars with mass $=30 \mathrm{M}_{\odot}$.

Table 9. Cluster mass of region A.

$\begin{array}{ccc} & \text { mass } / \mathrm{M}_{\odot} & \mathrm{N} \text { of stars } \\ 4.6(\mathrm{Myr}) & 3.110^{5} & 1.110^{5} \\ 14.5(\mathrm{Myr}) & 7.010^{5} & 2.710^{5} \\ 12<M<40 & 5.910^{4} & 3.110^{3} \\ 29<M<40 & 1.310^{4} & 4.010^{2} \\ 50<M<55(\mathrm{WR}) & 3.510^{3} & 59\end{array}$

The lower three rows give the mass intervals (in $\mathbf{M}_{\odot}$ ) of the young component.

This simplified two-bursts scenario is not appropriate for the nucleus or region $\mathrm{L}$, where the data are contaminated by the bulge light.

\section{DISCUSSION AND CONCLUSIONS}

The chemical composition of the gas has been obtained for the nucleus, four circumnuclear $\mathrm{H}$ il regions located inside a radius of $400 \mathrm{pc}$ centred at the nucleus, the 'Jumbo' region at 600 pc south-west from the centre and the $M$ region, which is located at 1600 pc north-west from the nucleus.

We found chemical abundances between 0.1 and 0.4 solar for the perinuclear, Jumbo and $\mathrm{M} \mathrm{H}$ II regions. The nuclear abundance is about solar. For this range of metallicities, the diagram $\log \left(\left[\mathrm{O}_{\mathrm{III}}\right] / \mathrm{H} \beta\right)$ versus $\log \left(\left[\mathrm{S}_{\mathrm{II}}\right] / \mathrm{H} \boldsymbol{\alpha}\right)$ is very sensitive to $U$ and to the ionizing temperature. From this diagram, we have found $\log U=-3$ for all the observed $\mathrm{H}_{\text {II }}$ regions (Fig 6a), the ionizing temperatures being in the range $37000<T_{\text {eff }}<40000 \mathrm{~K}$. The nuclear density is $8 \times 10^{3}$ $\mathrm{cm}^{-3}$, much higher than that of the other $\mathrm{H}$ in regions, which show normal $\mathrm{H}$ il region values. The observed line ratios for the nucleus are best fitted by models with $\log U=-3.2$, $T_{\text {eff }} \sim 40000 \mathrm{~K}$ and solar abundance.

Compared to other galaxies with circumnuclear $\mathrm{H}_{\text {II }}$ regions (NGC 1097, 2903, 2950, 5236 and 5248, KKB) NGC 3310 presents two important characteristics: (i) the $L_{\mathrm{H} \alpha}\left(\geq 4.0 \times 10^{39}\right)$ of all the observed regions are among the brightest observed hotspots (see fig. 2 in $\mathrm{KKB}$ ); region $\mathrm{C}$ is comparable to 30 Dor, and (ii) the metallicity in the perinuclear $\mathrm{H}$ II regions is notably smaller than that observed in other hotspot nuclei like NGC 1097, 1433, 7552, 1087 and $1408(12+\log \mathrm{O} / \mathrm{H} \leq 8.95$ and $12+\log \mathrm{N} / \mathrm{H} \leq 8.21$, Díaz 1985), which also have a Seyfert 2 or LINER nucleus.

We detected both WR and RSG (IR Ca II triplet) features in the spectrum of Jumbo, the most luminous of the observed
H II regions. The dominant WR types are WN4-5. The number of these stars is around 220 (60 Maeder's WN stars of 50-55 $\mathrm{M}_{\odot}$ will produce the same result). Since theoretical models predict that only stars with masses lower than $60 \mathrm{M}_{\odot}$ can reach the RSG phase, the presence of WN and RSG in an $\mathrm{H}$ il region with strong emission lines suggests an extended activity of star formation lasting more than a few million years.

A two-age model - 5 and $15 \mathrm{Myr}$ - for the starburst activity (equivalent to an extended star-formation activity) provides a good match to the observations. We also find that the emitted spectrum of the nucleus requires only photoionization by hot stars to be explained. The nuclear ionizing association (comparable to that in region $\mathrm{L}$ and five times smaller than that of the 'Jumbo' H il region) requires about $140 \mathrm{O} 6 \mathrm{~V}$ stars to explain the observed nuclear ionizing flux.

\section{ACKNOWLEDGMENTS}

The INT is operated on the island of La Palma by the RGO at the Observatorio del Roque de los Muchachos of the Instituto de Astrofísica de Canarias. This work has been partially supported by the British Council and the Brazilian Research Council CNPq. Fruitful discussions with Eduardo Bica, Charles Bonatto and Bernard Pagel are acknowledged. Gary Ferland kindly made available to us the latest version of CLOUDY. We thank CAT for awarding observing time.

\section{REFERENCES}

Aller L. H., 1984, Physics of Thermal Gaseous Nebulae. Reidel, Dordrecht

Armus L., Heckman T. M., Miley G. K., 1988, ApJ, 326, L45

Arp H., 1966, Atlas of peculiar galaxies. CalTech, Pasadena

Bertola F., Sharp N. A., 1984, MNRAS, 207, 47

Bottinelli L., Gouguenheim L., Paturel G., de Vaucouleurs G., 1984, A\&A, 56, 381

Brocklehurst M., 1971, MNRAS, 153, 471

Campbell A. W., Smith L. J., 1986, in De Loore C. W. H., Willis A. J., Laskarides P., eds, Proc. IAU Symp. 116, Luminous Stars and Associations in Galaxies. Reidel, Dordrecht, p. 499.

Cid-Fernandes R., 1989, MSc. Thesis, IF-UFRGS, P. Alegre, Brazil

Cid-Fernandes R., Dottori H., Viegas S. M., Gruenwald R., 1992, in Edmunds M. G., Terlevich R., eds, 31 st Herstmonceux Conference, Elements and the Cosmos. Cambridge University Press, Cambridge, p. 253

Clegg R. E. S., Middlemass D., 1987, MNRAS, 228, 759

Copetti M. V., Pastoriza M., Dottori H., 1986, A\&A, 156, 111

de Vaucouleurs G., de Vaucouleurs A., Corwin H. H., 1976, Second Reference Catalogue of Bright Galaxies. University of Texas, Austin

Díaz A. I., 1985, PhD Thesis, University of Sussex

Díaz A. I., Pagel B. E. J., Wilson I. G., 1985, MNRAS, 212, 737

Durret F., Bergeron J., Boksenberg A., 1985, A\&A, 143, 347

Evans I. N., Dopita M. A., 1985, ApJS, 57, 1

Ferland G., 1989, Internal Report 89-001. Astronomy Dept, Ohio State University

Grothues H. G., Schmidt-Kaler Th., 1991, A\&A, 242, 357; GSK91

Heckman T. M., Balick B., 1980, A\&A, 83, 100

Kennicutt R., Keel W., Blaha C., 1989, AJ, 97, 1022; KKB

Kunth D., Sargent W. L., 1981, A\&A, 101, L1; KS81

Kurucz R. L., 1979, ApJS, 40, 1

Lundström I., Stenholm B., 1984, A\&AS, 56, 43

Maeder A., 1990, A\&AS, 84, 139

Maeder A., Meynet G., 1989, A\&A, 210, 155 
Melnick J., 1985, A\&A, 153, 235

Mendoza C., 1983, in Flower D. R., ed., Proc. IAU Symp. 103, Planetary Nebulae. Reidel, Dordrecht, p. 143

Mihalas D., 1972, Tech. Notes/STR-76, National Center Atmosph. Research, Boulder, Colorado

Norman C., Scoville N., 1988, ApJ, 332, 124

Oke J. B., Gunn J. E., 1983, ApJ, 266, 713

Osterbrock D. E., 1989, Astrophysics of Gaseous Nebulae and Active Galactic Nuclei. University Science Books, Mill Valley, California

Osterbrock D. E., Cohen R. D., 1982, ApJ, 261, 64

Schild H., Kunth D., 1986, A\&A, 169, 71; SK86

Seaquist E. R., Bignell R. C., 1977, A\&A, 55, 163

Seaton M. J., 1975, MNRAS, 170, 475

Sersic J. L., Pastoriza M. G., 1967, PASP, 79, 152

Smith L. F., Kuhi L. V., 1981, Report 17, Joint Institute for Lab. Astrophys., Boulder, Colorado; SK81

Stasińska G., 1982, A\&AS, 48, 299
Telesco C. M., Gatley I., 1984, ApJ, 284, 557

Terlevich E., Díaz A. I., Pastoriza M. G., Terlevich R., Dottori H., 1990, MNRAS, 242, 48P

Terlevich R., Melnick J., 1985, MNRAS, 213, 841

Terlevich R., Melnick J., Moles M., 1987, in Khachikyan E. Ye., Fricke K. J., Melnick J., eds, Observational Evidence for Activity in Galaxies. Reidel, Dordrecht, p. 499

van der Hucht K. A., Conti P., Lunström L., Stenholm B., 1981, Space Sci. Rev., 28, 227

van der Kruit P. C., 1976, A\&A, 49, 161

van der Kruit P. C., de Bruyn A. G., 1976, A\&A, 48, 373

Vorontsov-Velyaminov B. A., 1977, A\&AS, 28, 1

Walborn N. R., 1991, in Haynes R., Milne D., eds, The Magellanic Clouds. Kluwer, Dordrecht, p. 145

Walborn N. R., Ebbets D. C., Parker J. W., Nichols-Bohlin J., White R. L., 1992, ApJ, in press

Wesemael F., 1981, ApJS, 45, 177

Whitford A. E., 1958, AJ, 63, 201 